\title{
Acquisition d'une L2 : résultats fondamentaux et perspectives
}

\author{
Sandra Benazzo \\ Savoirs, Textes, Langage (STL) \\ sandra.benazzo@univ-lille3.fr
}

\begin{abstract}
Cette contribution abordera un certain nombre d'études concernant l'acquisition d'une langue seconde (L2) par l'apprenant adulte. Dans un premier moment il sera question de quelques points forts de la recherche dans ce domaine. En effet, la compréhension des étapes fondamentales de l'acquisition d'une L2 a considérablement progressé du moment où les lectes d'apprenants ont été considérés comme des systèmes à part entière, et non pas comme des tentatives plus ou mieux réussies d'imitation de la langue cible. Les études interlinguistiques, disposant de données comparables pour l'acquisition de différentes langues, ont montré également des tendances communes, indépendantes des langues en contact, où le parcours acquisitionnel peut être décrit comme la grammaticalisation progressive d'un système initialement constitué de moyens lexicaux.
\end{abstract}

L'attention sera ensuite centrée sur quelques recherches récentes et en cours. Il sera question en particulier de deux projets. Le premier (Scope Particles \& Information Structure) porte sur la structure informationnelle et choix de perspective en L2. A partir de données obtenues grâce à un même support, ce projet vise à détecter des préférences interlinguistiques dans l'encodage de certaines relations anaphoriques chez des apprenants avancés. Il s'agit de tester l'hypothèse selon laquelle à un stade avancé, voire quasi-natif, l'apprenant maîtrise la grammaire de la phrase mais continue à encoder les relations discursives suivant le biais rhétorique de sa langue maternelle.

Le deuxième projet (Restricted Linguistic Systems as Windows on Language Genesis) montre comment les études sur l'acquisition des langues peuvent contribuer au débat sur les origines du langage. Les systèmes linguistiques résultant de différents processus acquisitionnels présentent un répertoire et des principes organisationnels plus simples que ceux des langues conventionnelles. Etant donné l'absence de traces sur des formes primordiales du langage humain, nous partons de l'idée que l'étude comparée de processus acquisitionnels actuels peut nous aider à restreindre les hypothèses sur un phénomène qui n'est pas directement observable. 